\title{
Phenotype difference between ALS patients with expanded repeats in C9ORF72 and patients with mutations in other ALS-related genes
}

Stéphanie Millecamps ${ }^{1 *}$, Séverine Boillée ${ }^{1}$, Isabelle Le Ber ${ }^{1}$, Danielle Seilhean ${ }^{1,2}$, Elisa Teyssou ${ }^{1}$, Marine Giraudeau 1 , Carine Moigneu${ }^{1}$, Nadia Vandenberghe ${ }^{3}$, Véronique Danel-Brunaud ${ }^{4}$, Philippe Corcia $^{5}$, Pierre-François Pradat6, Nadine Le Forestier ${ }^{6}$, Lucette Lacomblez ${ }^{6,7}$, Gaelle Bruneteau ${ }^{6}$, William Camu ${ }^{8}$, Alexis Brice ${ }^{9}$, Cécile Cazeneuve ${ }^{9}$, Eric LeGuern ${ }^{1,9}$, Vincent Meininger ${ }^{6}$, and François Salachas 6

${ }^{1}$ Centre de Recherche de l'Institut du Cerveau et de la Moelle Epinière, INSERM UMR_S975, CNRS UMR7225, Université Pierre et Marie Curie-Paris 6, Hôpital Pitié-Salpêtrière, Paris, France.

2Département de Neuropathologie, AP-HP, Hôpital Pitié-Salpêtrière, Paris, France.

${ }^{3}$ Hospices Civils de Lyon, Hôpital Neurologique Pierre Wertheimer, Bron, France

${ }^{4}$ Service de Neurologie et Pathologie du Mouvement, Hôpital Roger Salengro, CHRU Lille, France.

${ }^{5}$ Centre SLA, CHU de Tours, Université François Rabelais, Tours, France

${ }^{6}$ Fédération des Maladies du Système Nerveux, APHP, Centre de référence maladies rares SLA, Hôpital PitiéSalpêtrière, Paris, France.

7Département de Pharmacologie, INSERM UMR S678, Université Pierre et Marie Curie-Paris 6, Hôpital PitiéSalpêtrière, Paris, France.

${ }^{8}$ Service de Neurologie, Hôpital Guy de Chauliac, Université de Montpellier I, Montpellier France

${ }^{9}$ Assistance Publique Hôpitaux de Paris (AP-HP), Département de Génétique et Cytogénétique, Unité Fonctionnelle de neurogénétique moléculaire et cellulaire, Hôpital Pitié-Salpêtrière, Paris, France.

"To whom correspondence should be addressed: Dr Stéphanie Millecamps, Centre de Recherche de l'Institut du Cerveau et de la Moelle épinière, Groupe hospitalier PitiéSalpêtrière, 83, Bd de l'Hôpital, 75013 Paris, France, tel: 33157274341, e-mail: stephanie.millecamps@upmc.fr

Title count: 129 characters including spaces

Word count: 2435

Abstract word count: 250

References: 18

Figures/Tables: 2 Figures, 2 tables and 2 supplemental figures 


\section{Licence for publication:}

The Corresponding Author has the right to grant on behalf of all authors and does grant on behalf of all authors, an exclusive licence (or non-exclusive for government employees) on a worldwide basis to the BMJ Publishing Group Ltd and its Licensees to permit this article (if accepted) to be published in Journal of Medical Genetics and any other BMJPGL products to exploit all subsidiary rights, as set out in our licence (http://group.bmi.com/products/journals/instructions-for-authors/licence-forms).

\section{Contributorship statement:}

Dr Millecamps designed the study, interpreted the data and wrote the paper.

Drs Millecamps and Meininger performed statistical analyses.

Drs Boillee, LeGuern, Meininger and Salachas revised the manuscript for important intellectual content. Mrs Teyssou, Giraudeau and Moigneu and Dr Cazeneuve analyzed the genetic data.

Drs Le Ber, Seilhean, Vandenberghe, Danel-Brunaud, Corcia, Le Forestier, Lacomblez, Bruneteau Camu, Brice, Meininger and Salachas acquired and analyzed the clinical data.

All authors of this paper have read and approved the final version of the manuscript. Dr Millecamps takes full responsibility for the data, the analyses and interpretation, and the conduct of the research.

Competing interests: none declared

Funding: Association pour la Recherche sur la Sclérose latérale amyotrophique et autres maladies du motoneurone (ARSla, France) and Association française contre les myopathies (AFM, France).

Patient consent: Obtained.

Ethics approval: This study was conducted with the approval of the "Comité d'Ethique de la PitiéSalpêtrière" and the Medical Research Ethics Committee of "Assistance Publique-Hôpitaux de Paris".

Key words: Motor neuron disease, familial ALS, Genetic analysis, repeat primed PCR, GGGGCC repeat 


\section{Abstract}

Background. Expanded GGGGCC hexanucleotide repeats in the promoter of the C9ORF72 gene have recently been identified in frontotemporal dementia (FTD), Amyotrophic Lateral Sclerosis (ALS) and ALS-FTD and appear as the most common genetic cause of familial (FALS) and sporadic (SALS) forms of these diseases.

Methods. We searched for the C9ORF72 repeat expansion in 950 French ALS patients (225 FALS and 725 SALS) and 580 control subjects and performed genotype-phenotype correlations.

Results. The repeat expansion was present in $46 \%$ of FALS, $8 \%$ of SALS and $0 \%$ of controls. Phenotype comparisons were made between FALS patients with expanded C9ORF72 repeats and patients carrying another ALS-related gene (SOD1, TARDBP, FUS) or a yet unidentified genetic defect. SALS patients with and without C9ORF72 repeat expansions were also compared. The C9ORF72 group presented more frequent bulbar onset both in FALS ( $p<0.0001$ vs SOD1, $p=0.002$ vs TARDBP, $p=0.011$ vs FUS, $p=0.0153$ vs other FALS) and SALS ( $p=0.047)$. FALS patients with C9ORF72 expansions had more frequent association with FTD than the other FALS patients ( $p<0.0001$ vs SOD1, $p=0.04$ vs TARDBP, $p=0.004$ vs FUS, $p=0.03$ vs other FALS). C9ORF72-linked FALS patients presented an older age of onset than SOD1 $(\mathrm{p}=0.0139)$ or FUS mutation $(\mathrm{p}<0.0001)$ carriers. Disease duration was shorter for C9ORF72 expansion carriers than for SOD1 $(\mathrm{p}<0.0001)$ and TARDBP $(p=0.0242)$ carriers, other FALS $(p<0.0001)$ and C9ORF72-negative SALS $(p=0.0006)$.

Conclusions. Our results confirm the major role of expanded repeats in C9ORF72 as causative for ALS and provide evidence for specific phenotypic aspects compared to patients with other ALS-related genes. 


\section{Introduction}

Amyotrophic Lateral Sclerosis (ALS) is a fatal adult onset motor neuron disease with familial transmission in $6-10 \%$ of the cases. Mutations in SOD1 encoding the cooper/zinc superoxide dismutase, TARDBP (TAR DNA-binding protein 43) and FUS (fused in sarcoma) occur in $20-30 \%$ of familial forms of the disease (FALS). ${ }^{[1]}$ Rare cases of ALS are also linked to mutations in ANG, VAPB, DAO, OPTN, VCP and UBQLN2.[1, 2, 3, 4, 5] As ANG encodes a pancreatic ribonuclease with regulatory functions on ribosomal RNA transcription and TARDBP and FUS encode proteins with putative similar DNA/RNA-binding functions, defects in RNA processing pathways are an appealing hypothesis for ALS disease. The recent discovery of expanded hexanucleotide repeats in a non coding region of C9ORF72 in ALS, frontotemporal dementia (FTD), and ALS-FTD $[6,7,8]$ supports this view since these repeats seem to lead to the downregulation of the expression an alternatively spliced C9ORF72 transcript and to the formation of nuclear RNA foci. ${ }^{[6,7]}$ In this study we analyzed 950 French ALS patients to evaluate the frequency of the repeat expansion in C9ORF72 in FALS and SALS, and compared clinical phenotypes shown by these patients to patients with mutations in other ALS related genes.

\section{Methods}

\section{Patients}

FALS included 225 index cases of unrelated families with probable or definite ALS [9], 131 males and 94 females (M:F ratio= 1.4:1) with mean age of onset at 55 years (SEM 1, median 55 years, range 2185 years,) and mean disease duration of 51 months (SEM 4, median 33 months, range 3-336 months, including 32 censored data). SALS included 725 patients, 420 males and 305 females (M:F ratio=1.4:1), with mean age of onset at 56 years (SEM 1, median 58 years, range 21-87 years) and mean disease duration of 72 months (SEM 4, median 48 months, range 1-354 months, including 90 censored data). Data were censored at the last date of the patients visit. Control samples were age-matched Caucasian individuals of French background ( $n=580$ ). Most DNA samples were collected over the past 15 years at 
the ALS National Referal Center of Pitié-Salpêtrière Hospital (Paris). During the same period, the number of ALS patients followed by the center was 7784 . Some families were collected by other French ALS Centers belonging to the French ALS study group. Criterion for family inclusion was that at least two members were affected. In FALS the disease was transmitted in a dominant $(n=164)$ or putatively recessive (all affected members belonged to a single sibling with none of the parent affected, $n=47$ ) manner. In 14 remaining families, the ALS patients were distant relatives (more than $2^{\text {nd }}$ degree relatives). All participants signed a consent form allowing performing research. Protocols were approved by the Medical Research Ethics Committees of the "Comité d'Ethique de la Pitié-Salpêtrière" and "Assistance Publique-Hôpitaux de Paris".

\section{Genetic analysis}

All FALS have previously been screened for SOD1, ANG, TARDBP, FUS, DAO and OPTN[10, 11, 12]. FALS without male-to-male transmission $(n=130)$ had also been analyzed for the X-linked UBQLN2 gene [13]. Patients with mutation in SOD1 $(n=26), \operatorname{ANG}(n=1), \operatorname{TARDBP}(n=8), \operatorname{FUS}(n=13), D A O(n=1)$, $\operatorname{OPTN}(n=2)$, UBQLN2 $(n=1)$ and with no previously identified genetic defect $(n=173)$ were included in the C9ORF72 repeat analysis. For each patient, sequencing of ALS related genes and determination of C9ORF72 repeat length were performed on the same DNA sample.

The analysis of the C9ORF72 repeat was performed by a repeat-primed PCR amplification as previously described.[6] This analysis was completed by a classical fluorescent fragment-length analysis allowing the detection of non-expanded C9ORF72 alleles.[6] Both analyses were repeated twice for each patient sample to ensure reproducibility of the results, determine whether the repeat expansion was present at the heterozygous or homozygous state and because repeat primed assay efficiency highly depends on DNA concentration and quality. DNA of some homozygote individuals was sequenced to precisely correlate the number of base pairs in the fluorescent assay with the number of GGGGCC repeats. For clinical comparison, only patients with GGGGCC repeat numbers greater than 50 were included in the C9ORF72 group. Although no biological data is available to support that 50 repeats is a 
suitable cut-off to determine pathogenicity, this 50 repeat cut-off corresponds to the detection limit of the method we used.

\section{Clinical analysis}

Clinical data could be partially recovered for up to 334 relatives belonging to the 225 analyzed families and 725 SALS cases. The exact number of patients included in each statistical analysis depended on the availability of the corresponding data and is summarized in Tables 1 and 2. Clinical parameters including gender ratio ( $n=334$ FALS, 719 SALS), age of onset ( $n=290$ FALS, 512 SALS), site of onset ( $\mathrm{n}=283$ FALS, 703 SALS), disease duration ( $\mathrm{n}=264$ FALS, 512 SALS) ${ }^{[10]}$ and presence of FTD behavioral variant ( $n=162$ FALS) ${ }^{[14]}$ were compared in 5 FALS groups represented by patients with long C9ORF72 repeat, mutations in SOD1, TARDBP, FUS or other FALS with still unidentified mutations (table 1). In addition, 2 groups of SALS patients either positive or negative for expanded C9ORF72 repeats were included. Disease duration was also compared between C9ORF72 patients with bulbar $(n=42)$ or spinal $(n=70)$ onset and with $(n=25)$ or without $(n=28)$ FTD. FALS were arbitrarily separated according to age of onset $(\leq 40,41-50,51-60,61-70, \geq 71)$ to study the frequency of ALS-related genes in each subgroup. Two consecutive subgroups were pooled when their distributions were similar.

\section{Statistical analysis}

Statistical analyses were performed as previously described.[10] Briefly, the Cox proportional hazards regression model was used to compare age at onset and disease duration between the five groups of FALS patients. If the difference was significant $(p<0.05)$, a log rank analysis compared groups by pairs. Proportions of patients classified according to gender, site of onset or presence of FTD were compared by pairs using Fisher's exact tests. Proportions of C9ORF72-positive subgroup of patients $(n=70)$ with available cognitive status data (FTD or pure ALS) classified according to site of onset (bulbar or spinal) were also compared using Fisher's exact test. Statistical analyses were performed using the SPSS 11.0 data analysis software (SPSS Inc). 


\section{Results}

In controls, the mean number of C9ORF72 repeat expansions was of 4 (range $=2-23$ ) and no repeat expansion greater than 23 was detected whereas a repeat length higher than 23 was identified at the heterozygous state in 104 FALS (46\%) and 57 SALS (8\%) (supplementary figure 1). The frequency of the $16-23$ repeat alleles was similar in ALS patients (2.9\%) and control subjects (2.8\%). We detected one FALS and four SALS patients with 24 repeats and two other SALS patients with 25 repeats. These intermediate repeat length carriers were not included in clinical comparison analysis. All the other positive ALS patients had GGGGCC repeat numbers greater than 50 corresponding to the detection limit of the method we used. The frequency of SALS with expansion length of more than 50 repeats was of $7 \%$.

The segregation of the expanded repeat with the disease could be confirmed in 16 families (supplementary figure 2). The pedigrees showed that some obligate carriers were asymptomatic. Although in several families their age at death could not be determined, one of them died at 88 .

The contribution of C9ORF72 gene in FALS varied according to age at disease onset: it was detected in only $17 \%$ of FALS reaching disease onset before 40 years of age (where FUS mutations are the majority) and in more than $50 \%$ of FALS with disease onset starting after 40 (figure $1 \mathrm{~A}$ ). The two groups corresponding to onset age between 41 and 60 or $>61$ held similar rates of $51 \%$ and $52 \%$ respectively. Age at onset for the C9ORF72, SOD1, TARDBP, FUS and other FALS groups was statistically different (Cox regression test: $p<0.001$, figure 1D). C9ORF72 patients presented onset later than SOD1 (log rank: $p=0.0139$ ) and FUS patients (log rank: $p<0.0001)$ whereas no statistically significant difference was found with the TARDBP or other FALS groups. In SALS, no difference was observed between age at onset of C9ORF72-positive and -negative patients (figure 2B).

The proportion of FALS with bulbar, lower limb and upper limb onset was different between C9ORF72 patients and the other groups (figure $1 \mathrm{~B}$, table 1). The site of onset was heterogeneous for C9ORF72 
FALS patients, with more frequent bulbar onset (40\%) than in SOD1 (7\%), TARDBP (11\%), FUS (14\%) or other FALS (20\%) patients (figure 1B). In SALS, C9ORF72 patients had also more frequent bulbar onset $(40 \%)$ than the remaining ones $(25 \%)$ (figure $2 \mathrm{~A})$.

Information about FTD was available only for a minority of our patients (figure 1C, table 1) but showed that it was more frequent in C9ORF72 FALS patients (40\%) than in SOD1 (0\%), TARDBP (17\%), FUS $(5 \%)$ and other FALS (17\%) patients. A conclusion about FTD in SALS could not be drown since the information could be recovered for only $16 \%$ of the patients. The proportion of C9ORF72 FALS patients with bulbar onset was similar (41\%) whether they presented or not FTD.

Disease duration differed in the 5 groups of FALS patients (Cox regression test: $p<0.001$, figure 1E). It was shorter for C9ORF72 patients than for SOD1 (log rank, $\mathrm{p}<0.0001$ ), TARDBP (log rank, $\mathrm{p}=0.0242$ ) and other FALS (log rank, p<0.0001) patients and similar to the FUS group (figure 1E). In SALS, disease duration was shorter for C9ORF72 patients than for the other SALS (log rank, $\mathrm{p}=0.0006$; figure 2C). In C9ORF72 FALS patients, disease duration was shorter when presenting bulbar onset compared to spinal onset (log rank, $p=0.04$; figure $1 \mathrm{~F}$ ) but was not statistically different between patients affected or not by FDT. No gender effect was observed between C9ORF72 FALS patients and the 4 other groups of FALS, and between C9ORF72 SALS patients compared to the remaining SALS (tables 1-2).

Expanded C9ORF72 repeats were also found in our patients carrying a mutation in ANG (c.122A>T, pLys41lle), DAO (c.113G>A, p.Arg38His), OPTN (c.382_383insAG, p.Asp128GlufsX22) or UBQLN2 (c.1500_1508delCATAGGCCC, p.Gly502_Ile504del) whom the pathogeneicity of was questioned in previous studies. $\left.{ }^{10,11} 112,13\right]$ The segregation of C9ORF72 expansion could be confirmed for the family with the UBQLN2 deletion that we previously showed not to segregate with ALS phenotype (supplementary figure 2P).[13] Two FALS with expanded C9ORF72 repeat (>50 repeats) also carried either a SOD1 (c.328G>T, p.Asp110Tyr, one out of the 26 index cases carrying a SOD1 mutation) or a FUS (c. 1561C>T, p.Arg521Cys, one out of 13 index cases carrying a FUS mutation) mutation. Since no 
other affected relative was collected in these two families, the segregation of the mutations could not be further studied.

The SOD1 (p.Asp110Tyr)-C9ORF72 repeat expansion patient had upper limb onset at 59 years of age with a 42-month-disease duration. The FUS (p.Arg521Cys)-C9ORF72 repeat expansion patient had a bulbar onset at 40 and disease duration of 14 months. These patients were not included in clinical comparison analyses.

\section{Discussion}

Our results confirm that the expanded repeat in C9ORF72 is the most common genetic defect in French ALS occurring in $46 \%$ of FALS and $8 \%$ of SALS. These frequencies of C9ORF72 expanded repeats were in the range of those previously reported for FALS (23-47\%) and SALS (4-21\%) from Belgium, Finland and the USA.[6, 7, 8] C9ORF72 repeat expansions were more frequent (50\% of FALS) in ALS patients with disease onset starting after 40 years of age whereas FUS mutations remained the most common genetic defect $(35 \%)$ in ALS patients with early onset ( $\leq 40$ years). Repeat length in our control group ranged from 2 to 23 , close to the findings of previous reports. [6,7,8]

Some obligate carriers were asymptomatic, suggesting either that the penetrance of the phenotype associated with the C9ORF72 expanded repeats was incomplete, or that the obligate carriers did not survive long enough to start the disease. Concerning SALS, the detection of C9ORF72 repeat expansions in these patients could be related to possible incomplete penetrance in their family, lack of complete family history or absence of clinical data regarding the dementia status of the relatives.

Comparing the phenotypes of FALS with expanded C9ORF72 repeats (> 50 repeats) to the ones of patients carrying a mutation in one of the other ALS-related genes (SOD1, TARDBP, FUS) and patients with unidentified genetic defects (other FALS) we found that C9ORF72 patients had a rather late age of onset (compared to patients with other identified genetic defects). They also had more frequent bulbar onset, more frequent associated FTD and shorter disease duration than the other groups, in agreement 
with two other recently reported studies on smallest cohorts of patients.[15, 16] The proportion of C9ORF72 FALS patients with bulbar onset was similar whether they presented or not FTD implying that the site of onset and the presence of cognitive impairment did not seem to be correlated. The shorter disease duration of the C9ORF72 carrier group could be related to the more frequent bulbar presentation of the disease observed in these patients. In SALS we confirmed that C9ORF72 expanded repeat carriers had more frequent bulbar onset and shorter disease duration.

We have also found C9ORF72 repeat expansions in patients with variants in other ALS-related genes. Thus ANG, DAO, OPTN and UBQLN2 genes appear now as negative in our cohort of 225 French FALS and should be considered as very rare causes of ALS. For patients in whom a C9ORF72 mutation was identified in addition to a potential pathogenic SOD1 mutation (SOD1 variant is an unreported substitution affecting an aminoacid that is not conserved among species) and a clearly pathogenic FUS mutation (p.Arg521Cys is one of the most frequent FUS mutation identified in ALS patients[17, 18]), we could not perform southern blot experiments due to limited amount of DNA for these deceased patients. As the repeat length could not be determined exactly in these patients, it is not possible to conclude if the expansion is as long as the previously reported expansions of 700 to 1600 repeat units (that were observed using southern blot analysis[6]) or if in these cases, the expansion was shorter. In any case, our results suggest that the pathogenicity of any novel SOD1 missense variant should be evaluated with caution.

Since this study points out phenotype-genotype correlations between FALS groups with different ALSrelated mutations, further analyses are required to define more precisely the number of repeats and to determine whether it is correlated with the severity of the phenotype (age of disease onset or disease duration) and/or the association of ALS with cognitive impairments including FTD.

Although our data have to be confirmed in other cohorts of patients, they confirm the major role of expanded repeats in C9ORF72 as causing ALS and provide evidence that these patients have a characteristic phenotype as compared to patients carrying other ALS related-gene mutations. In view of 
the frequency of this repeat expansion, the molecular diagnosis of ALS should be centered on the identification of this mutation.

\section{Legends of the figures}

\section{Figure 1. Clinical comparison of C9ORF72, SOD1, TARDPB, FUS and other FALS patients}

(A) Proportion of FALS patients with C9ORF72 repeat expansions (red), or with a mutation in SOD1 (green), FUS (yellow) and TARDBP (blue), or with a still unidentified genetic cause (Other FALS, black) are presented according to age of onset: before $40 \mathrm{y}, 41-60 \mathrm{y}$, after $61 \mathrm{y}$.

$(B, C)$ Histograms showing the distribution of FALS patients with SOD1, TARDBP, FUS mutations, C9ORF72 expanded repeats or with unidentified genetic defect (other FALS) according to bulbar (black), upper limb (dark-gray) or lower limb (light gray) onset (B) and presence (black) or absence (gray) of FTD (C). A Fischer's exact test showed a statistically significant difference between the C9ORF72 group and all the other groups of patients.

Cox regression curves of cumulative probability of disease onset according to the age of FALS patients (D), and cumulative survival probability from time at disease onset (E) for C9ORF72 (black bold dotted line), FUS (black thin line), TARDBP (grey bold dotted line), SOD1 (grey thin dotted line), and other FALS (grey thin line) groups of patients. The survival curves of C9ORF72 and FUS and those of TARDBP and other FALS are superimposed. C9ORF72 and FUS patients had the shortest disease duration. Cox regression curve of cumulative survival probability from time at disease onset for C9ORF72 FALS patient with bulbar (black bold line) or spinal (black thin dotted line) onset (F). Note that disease duration was more rapid for C9ORF72 patients with bulbar onset. 


\section{Figure 2. Clinical comparison of SALS with or without C9ORF72 expanded repeats}

(A) Histograms showing the distribution of SALS patients with (C9ORF72) or without (other SALS) C9ORF72 expanded repeats according to bulbar (black) or spinal (gray) onset. A Fischer's exact test showed a statistically significant difference between the two groups of patients. Cox regression curves of cumulative probability of disease onset according to the age (B) and cumulative survival probability from time at disease onset $(C)$ for SALS patients who were positive (black bold dotted line) or negative (grey thin line) for C9ORF72 expanded repeats. Both curves showing probability of disease onset are superimposed. C9ORF72 patients had shorter disease duration than the other SALS.

\section{Supplementary figure 1. Analysis of the C9ORF72 GGGGCC hexanucleotide repeats in three ALS}

\section{patients.}

Repeat-primed PCR was used to detect expanded GGGGCC hexanucleotide (A-C). The reverse primer designed on the repeat sequence allowed the amplification of multiple fluorescent PCR products that were visualized using GeneMapper software v4.0. Examples of expanded C9ORF72 repeats (Ex) are shown by the typical 6 bp periodicity profile in A and B.

Fluorescent fragment length analyses of a PCR fragment containing the hexanucleotide repeats $\left(A^{\prime}-C^{\prime}\right)$. The reverse primer designed distantly to the repeats allowed to amplify PCR product fragments with limited numbers of repeats (2 in A', 6 in B', 2 and 8 in C'). Fluorescent PCR products were visualized using Peak Scanner software v1.0. The red line shows the GeneScan 500 ROX Size standard (Applied Biosystems). Note that the expanded repeats were not amplified in B and D. Genotypes of the patients deduced from these two analyses are presented in brackets. Patient 1 and 2 carried an expanded C9ORF72 repeat. Patient 3 had no expanded C9ORF72 repeat. 


\section{Supplementary figure 2. C9ORF72 repeat expansions segregate with the disease in 16 French}

\section{pedigrees.}

Abbreviated pedigrees of families with C9ORF72 expanded repeats in which the segregation of the repeat could be studied (A-P). Arrows indicate index patients. Filled symbols indicate patients with ALS and half-filled symbols represent ALS patients with concomitant FTD. When available, the age at onset (years), site at onset ( $B=$ bulbar, UL=Upper Limb, LL=Lower Limb, in brackets) and the disease duration (in months, in brackets) are indicated below the symbol representing the patient. The number of repeats is indicated for each allele below the index case and some relatives for whom DNA was available. Ex ("expanded repeat") represents a number of repeats $>50$. The age at death (88y) is indicated in brackets above an obligate carrier who did not develop the disease $(G)$.

\section{Acknowledgments}

We are grateful to the patients and their families. We thank the Généthon cell and DNA bank (Evry, France) and the CRicm DNA and cell bank (Paris, France) for patients' DNA. This work was financed by the Association pour la Recherche sur la Sclérose latérale amyotrophique et autres maladies du motoneurone (ARSla, France) and by Association française contre les myopathies (AFM, France).

\section{References}

1 Andersen PM, Al-Chalabi A. Clinical genetics of amyotrophic lateral sclerosis: what do we really know? Nat Rev Neurol 2011;7(11):603-15.

2 Mitchell J, Paul P, Chen HJ, Morris A, Payling M, Falchi M, Habgood J, Panoutsou S, Winkler S, Tisato V, Hajitou A, Smith B, Vance C, Shaw C, Mazarakis ND, de Belleroche J. Familial amyotrophic lateral sclerosis is associated with a mutation in D-amino acid oxidase. Proc Natl Acad Sci U S A 2010;107(16):7556-61.

3 Maruyama H, Morino H, Ito H, Izumi $\mathrm{Y}$, Kato H, Watanabe $\mathrm{Y}$, Kinoshita $\mathrm{Y}$, Kamada M, Nodera $H$, Suzuki $H$, Komure O, Matsuura S, Kobatake K, Morimoto N, Abe K, Suzuki N, Aoki M, Kawata A, Hirai T, Kato T, Ogasawara K, Hirano A, Takumi T, Kusaka H, Hagiwara K, Kaji R, Kawakami $\mathrm{H}$. Mutations of optineurin in amyotrophic lateral sclerosis. Nature 2010;465(7295):223-6. 
4 Jonsson PA, Graffmo KS, Brannstrom T, Nilsson P, Andersen PM, Marklund SL. Motor neuron disease in mice expressing the wild type-like D90A mutant superoxide dismutase-1. J Neuropathol Exp Neurol 2006;65(12):1126-36.

5 Deng HX, Chen W, Hong ST, Boycott KM, Gorrie GH, Siddique N, Yang Y, Fecto F, Shi Y, Zhai $\mathrm{H}$, Jiang H, Hirano M, Rampersaud E, Jansen GH, Donkervoort S, Bigio EH, Brooks BR, Ajroud K, Sufit RL, Haines JL, Mugnaini E, Pericak-Vance MA, Siddique T. Mutations in UBQLN2 cause dominant $X$-linked juvenile and adult-onset ALS and ALS/dementia. Nature 2011;477(7363):211-5.

6 Dejesus-Hernandez M, Mackenzie IR, Boeve BF, Boxer AL, Baker M, Rutherford NJ, Nicholson AM, Finch NA, Flynn H, Adamson J, Kouri N, Wojtas A, Sengdy P, Hsiung GY, Karydas A, Seeley WW, Josephs KA, Coppola G, Geschwind DH, Wszolek ZK, Feldman H, Knopman DS, Petersen RC, Miller BL, Dickson DW, Boylan KB, Graff-Radford NR, Rademakers R. Expanded GGGGCC Hexanucleotide Repeat in Noncoding Region of C9ORF72 Causes Chromosome 9pLinked FTD and ALS. Neuron 2011;72(2):245-56.

7 Renton AE, Majounie E, Waite A, Simon-Sanchez J, Rollinson S, Gibbs JR, Schymick JC, Laaksovirta H, van Swieten JC, Myllykangas L, Kalimo H, Paetau A, Abramzon Y, Remes AM, Kaganovich A, Scholz SW, Duckworth J, Ding J, Harmer DW, Hernandez DG, Johnson JO, Mok K, Ryten M, Trabzuni D, Guerreiro RJ, Orrell RW, Neal J, Murray A, Pearson J, Jansen IE, Sondervan D, Seelaar H, Blake D, Young K, Halliwell N, Callister JB, Toulson G, Richardson A, Gerhard A, Snowden J, Mann D, Neary D, Nalls MA, Peuralinna T, Jansson L, Isoviita VM, Kaivorinne AL, Holtta-Vuori M, Ikonen E, Sulkava R, Benatar M, Wuu J, Chio A, Restagno G, Borghero G, Sabatelli M, Heckerman D, Rogaeva E, Zinman L, Rothstein JD, Sendtner M, Drepper C, Eichler EE, Alkan C, Abdullaev Z, Pack SD, Dutra A, Pak E, Hardy J, Singleton A, Williams NM, Heutink P, Pickering-Brown S, Morris HR, Tienari PJ, Traynor BJ. A Hexanucleotide Repeat Expansion in C9ORF72 is the Cause of Chromosome 9p21-Linked ALS-FTD. Neuron 2011;72(2):257-68.

8 Gijselinck I, Van Langenhove T, van der Zee J, Sleegers K, Philjens S, Kleinberger G, Janssens J, Bettens K, Van Cauwenberghe C, Pereson S, Engelborghs S, Sieben A, De Jonghe P, Vandenberghe R, Santens P, De Bleecker J, Maes G, Baumer V, Dillen L, Joris G, Cuijt I, Corsmit E, Elinck E, Van Dongen J, Vermeulen S, Van den Broeck M, Vaerenberg C, Mattheijssens M, Peeters K, Robberecht W, Cras P, Martin JJ, De Deyn PP, Cruts M, Van Broeckhoven C. A C9orf72 promoter repeat expansion in a Flanders-Belgian cohort with disorders of the frontotemporal lobar degeneration-amyotrophic lateral sclerosis spectrum: a gene identification study. Lancet Neurol 2011.

9 Brooks BR, Miller RG, Swash M, Munsat TL. El Escorial revisited: revised criteria for the diagnosis of amyotrophic lateral sclerosis. Amyotroph Lateral Scler Other Motor Neuron Disord 2000;1(5):293-9.

10 Millecamps S, Salachas F, Cazeneuve C, Gordon P, Bricka B, Camuzat A, Guillot-Noel L, Russaouen O, Bruneteau G, Pradat PF, Le Forestier N, Vandenberghe N, Danel-Brunaud V, Guy N, Thauvin-Robinet C, Lacomblez L, Couratier P, Hannequin D, Seilhean D, Le Ber I, Corcia P, Camu W, Brice A, Rouleau G, Leguern E, Meininger V. SOD1, ANG, VAPB, TARDBP, and FUS mutations in familial amyotrophic lateral sclerosis: genotype-phenotype correlations. J Med Genet 2010;47(8):554-60.

11 Millecamps S, Da Barroca S, Cazeneuve C, Salachas F, Pradat PF, Danel-Brunaud V, Vandenberghe N, Lacomblez L, Le Forestier N, Bruneteau G, Camu W, Brice A, Meininger V, LeGuern $E$. Questioning on the role of $D$ amino acid oxidase in familial amyotrophic lateral sclerosis. Proc Natl Acad Sci U S A 2010;107(26):E107; author reply E8. 
12 Millecamps S, Boillee S, Chabrol E, Camu W, Cazeneuve C, Salachas F, Pradat PF, DanelBrunaud V, Vandenberghe N, Corcia P, Le Forestier N, Lacomblez L, Bruneteau G, Seilhean D, Brice A, Feingold J, Meininger V, LeGuern E. Screening of OPTN in French familial amyotrophic lateral sclerosis. Neurobiol Aging 2011;32(3):557 e11-3.

13 Millecamps S, Corcia P, Cazeneuve C, Boillee S, Seilhean D, Danel-Brunaud V, Vandenberghe N, Pradat PF, Le Forestier N, Lacomblez L, Bruneteau G, Camu W, Brice A, Meininger V, LeGuern E, Salachas F. Mutations in UBQLN2 are rare in French Amyotrophic Lateral Sclerosis Neurobiol Aging 2012;doi:10.1016/j.neurobiolaging.2011.11.010.

14 Neary D, Snowden JS, Gustafson L, Passant U, Stuss D, Black S, Freedman M, Kertesz A, Robert PH, Albert M, Boone K, Miller BL, Cummings J, Benson DF. Frontotemporal lobar degeneration: a consensus on clinical diagnostic criteria. Neurology 1998;51(6):1546-54.

15 Byrne S, Elamin M, Bede P, Shatunov A, Walsh C, Corr B, Heverin M, Jordan N, Kenna K, Lynch C, McLaughlin RL, Iyer PM, O'Brien C, Phukan J, Wynne B, Bokde AL, Bradley DG, Pender N, Al-Chalabi A, Hardiman O. Cognitive and clinical characteristics of patients with amyotrophic lateral sclerosis carrying a C9orf72 repeat expansion: a population-based cohort study. Lancet Neurol 2012; DOI 10.1016/S1474-4422(12)70014-5.

16 Stewart $\mathrm{H}$, Rutherford NJ, Briemberg H, Krieger C, Cashman N, Fabros M, Baker M, Fok A, Dejesus-Hernandez M, Eisen A, Rademakers R, Mackenzie IR. Clinical and pathological features of amyotrophic lateral sclerosis caused by mutation in the C9ORF72 gene on chromosome 9p. Acta Neuropathol 2012; DOI 10.1007/s00401-011-0937-5;

17 Kwiatkowski TJ, Jr., Bosco DA, Leclerc AL, Tamrazian E, Vanderburg CR, Russ C, Davis A, Gilchrist J, Kasarskis EJ, Munsat T, Valdmanis P, Rouleau GA, Hosler BA, Cortelli P, de Jong PJ, Yoshinaga Y, Haines JL, Pericak-Vance MA, Yan J, Ticozzi N, Siddique T, McKenna-Yasek D, Sapp PC, Horvitz HR, Landers JE, Brown RH, Jr. Mutations in the FUS/TLS gene on chromosome 16 cause familial amyotrophic lateral sclerosis. Science 2009;323(5918):1205-8.

18 Vance C, Rogelj B, Hortobagyi T, De Vos KJ, Nishimura AL, Sreedharan J, Hu X, Smith B, Ruddy D, Wright P, Ganesalingam J, Williams KL, Tripathi V, Al-Saraj S, Al-Chalabi A, Leigh PN, Blair IP, Nicholson G, de Belleroche J, Gallo JM, Miller CC, Shaw CE. Mutations in FUS, an RNA processing protein, cause familial amyotrophic lateral sclerosis type 6. Science 2009;323(5918):1208-11. 
$\begin{array}{llllll}\text { Patient groups } & \text { C9ORF72 } & \text { SOD1 } & \text { TARDBP } & \text { FUS } & \text { other FALS }\end{array}$ Age at Onset (years)

Mean

58 (SE 1)

51 (SE 2)

52 (SE 3)

43 (SE 2)

60 (SE 2)

Median

57

51

57

40

61

Range

$30-81(n=120)$

$21-73(n=46)$

$27-78(n=18)$

$25-68(n=36) \quad 32-85(n=70)$

\section{Disease duration (months) *}

Mean

33 (SE 2)

81 (SE 12)

56 (SE 8)

34 (SE 4)

66 (SE 12)

Median 29

49

48

28

39

Range

$3-85(n=112)$

$4-252(n=44)$

$20-156(n=18)$

$9-88(n=32)$

$8-336(n=58)$

\section{Bulbar onset **}

Ratio of patients

$48 / 120$

$3 / 41$

2/18

$5 / 35$

$14 / 69$

FTD ***

Ratio of patients

$27 / 67$

$0 / 27$

$2 / 18$

$1 / 20$

$5 / 30$

\section{Gender}

M:F ratio

$1.5: 1(n=130)$

$2.1: 1(n=81)$

$1.2: 1(n=56)$

$1: 1.7(n=27)$

1.1:1 $(n=40)$

table 1. Clinical comparison between groups of patients with a mutation in, SOD1, TARDBP, FUS, hexanucleotide repeats in C9ORF72 and patients with no identified genetic defect (other FALS)

$\mathrm{SE}=$ standard error. $\mathrm{n}=$ number of patients with the information available.

*: disease duration data included censored data (11 for C9ORF72, 8 for SOD1, 1 for TARDBP, 1 for FUS and 11 for other FALS).

${ }^{* *}$ : the fraction represents the number of patients with bulbar onset / the total number of patients for whom site of onset information was available

***: the fraction represents the number of patients with frontotemporal dementia (FTD) / the total number of patients who were examined for cognitive deficits. 
Patient groups

Age at Onset (years)

Mean

Median

Range

Disease duration (months) *

Mean

Median

Range

\section{Bulbar onset **}

Ratio of patients

$20 / 51$

$167 / 652$

Gender

M:F ratio

$1.55: 1(n=51) \quad 1.4: 1(n=668)$

$35-77(n=31) \quad 21-87(n=481)$

38 (SE 4) $\quad 73$ (SE 4)

$37 \quad 48$

$9-133(n=31) \quad 1-354(n=481)$ other SALS

56 (SE 1)

58

table 2. Clinical comparison between groups of SALS patients with or without expanded repeats in C9ORF72.

$\mathrm{SE}=$ standard error. $\mathrm{n}=$ number of patients for whom the information was available.

*: disease duration data included censored data ( 3 for C9ORF72 and 87 for other SALS).

${ }^{* *}$ : the fraction represents the number of patients with bulbar onset / the total number of patients for whom site of onset information was available. 


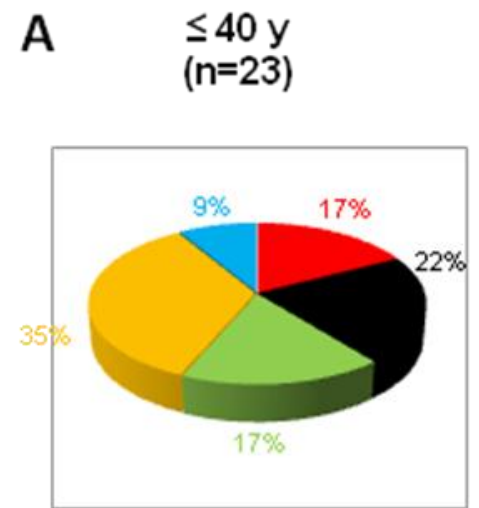

B

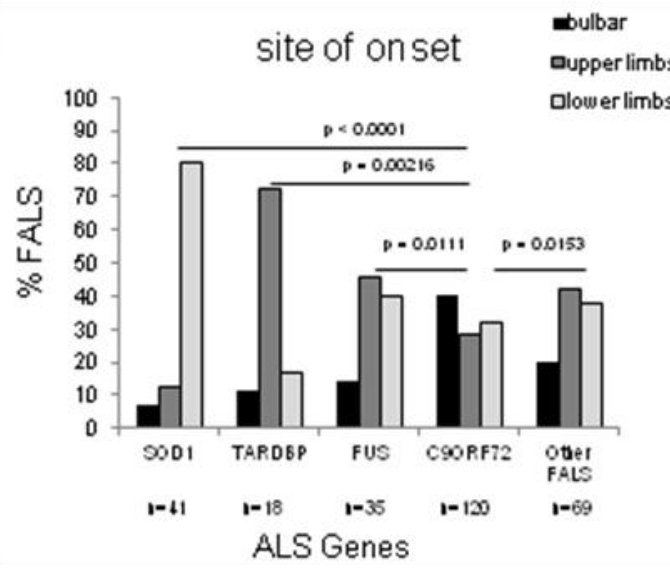

( $n=113$ )
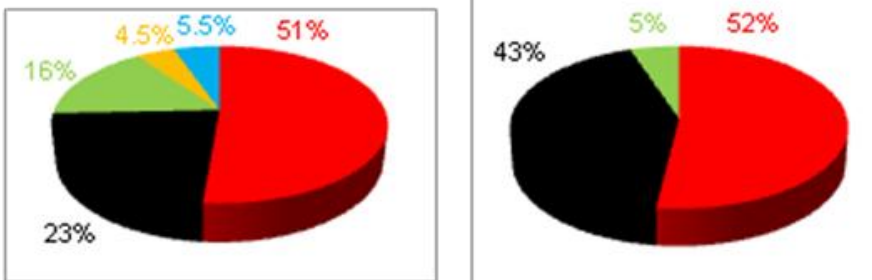

- C9ORF 72

-Other F ALS

$=$ SOD1

aFUS

-TARDBP $\geq 61 y$

( $\mathrm{n}=77$ )
D

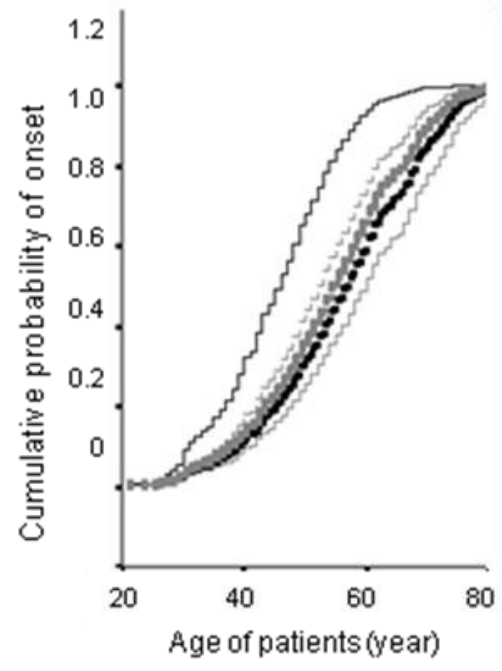

E

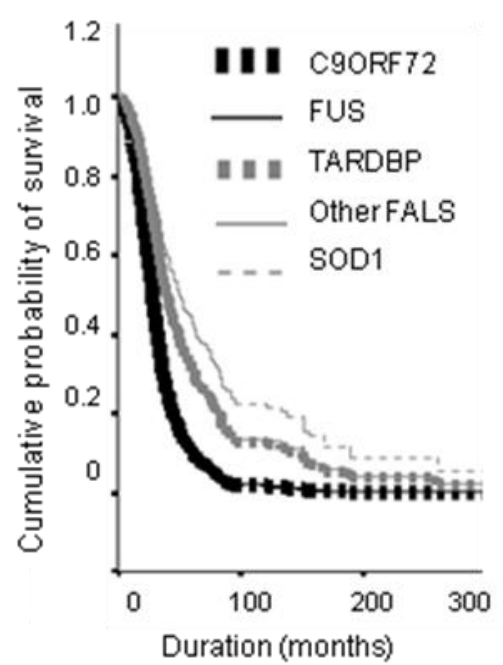

F

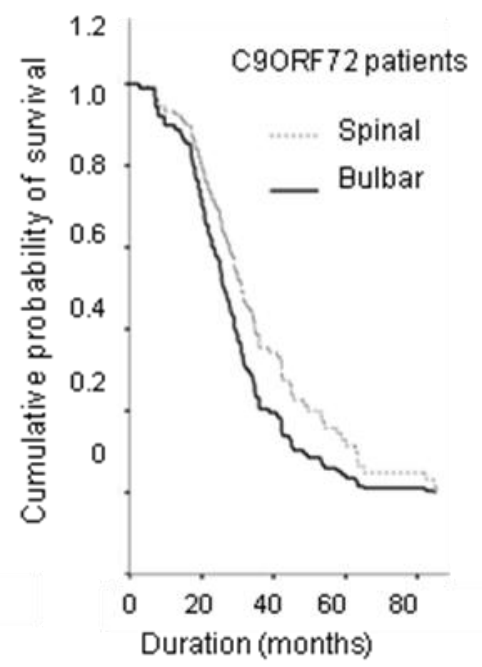

Fig.1 
A

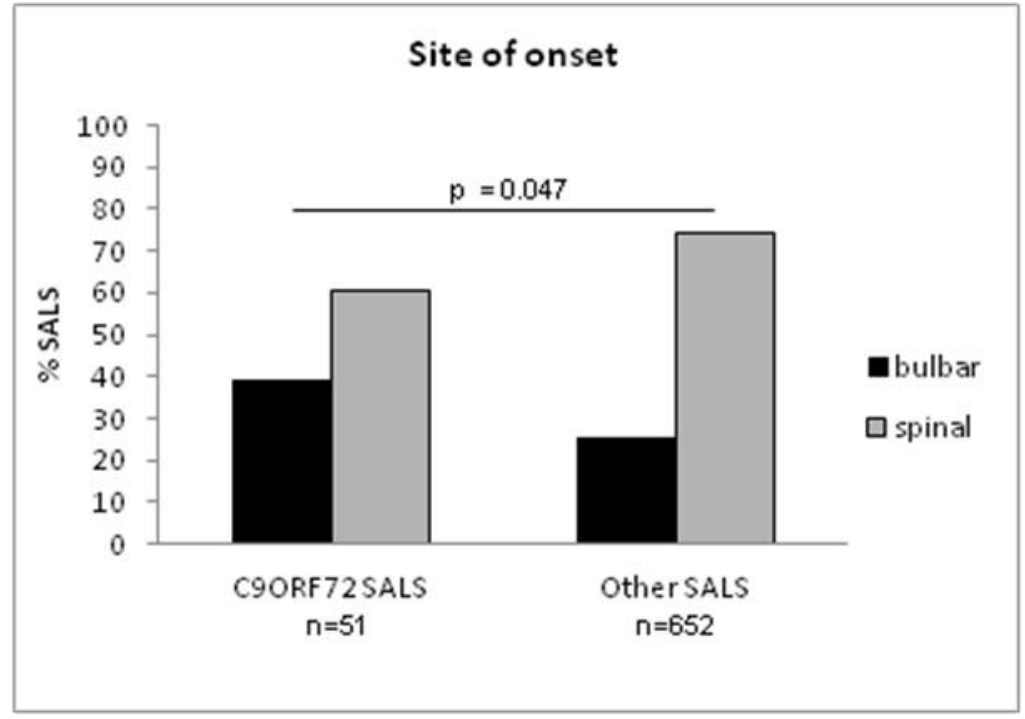

B

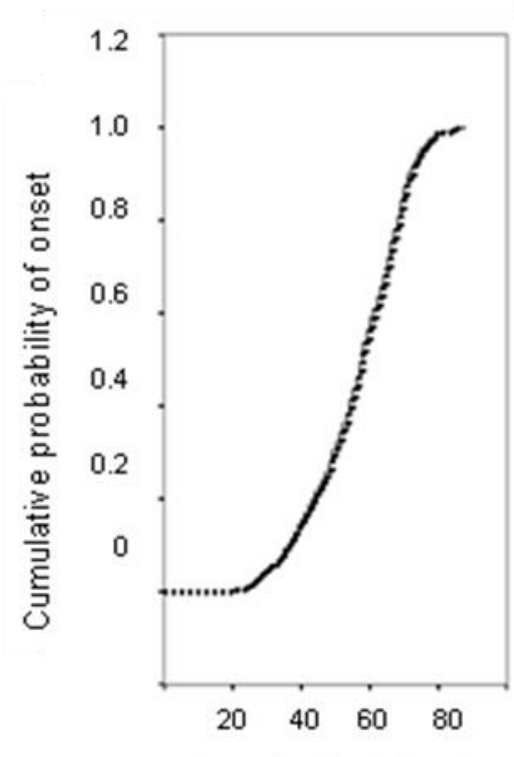

Age of patients (year)
C

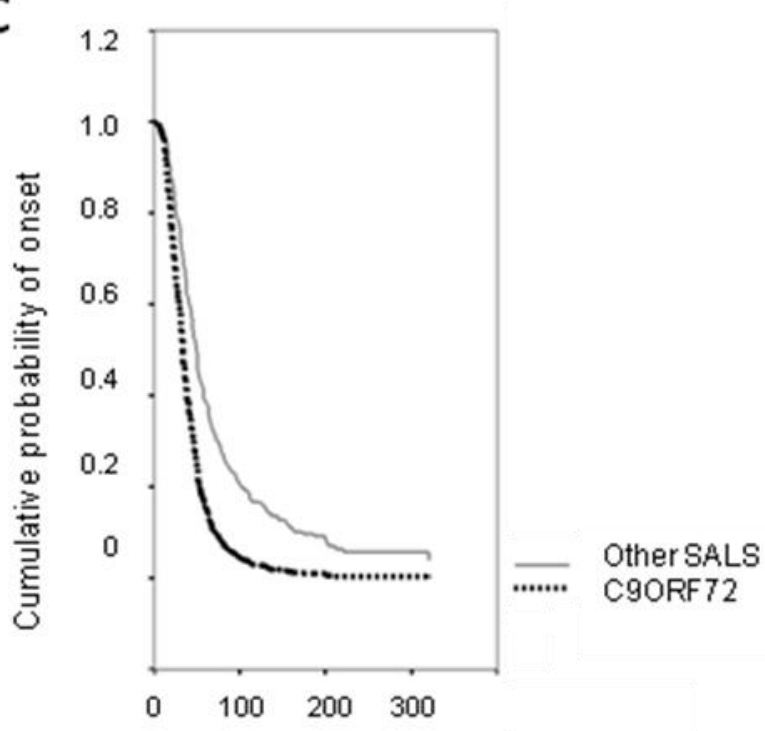

Duration (months)

Fig. 2 


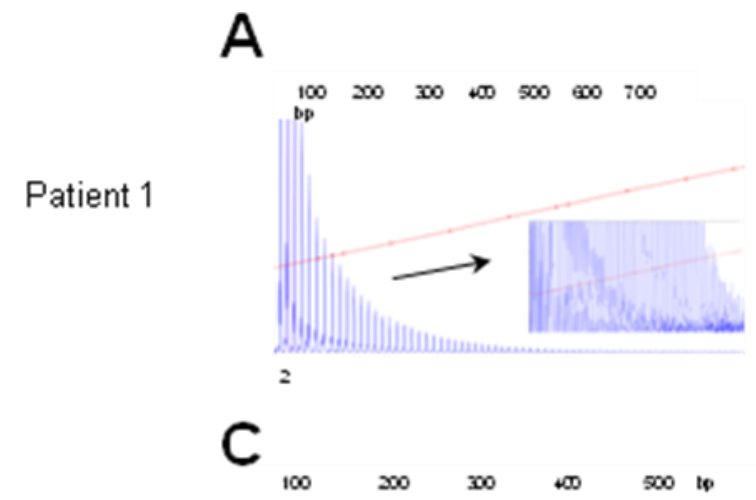

B

Patient 2

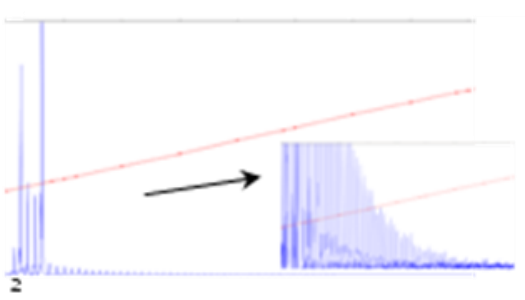

E

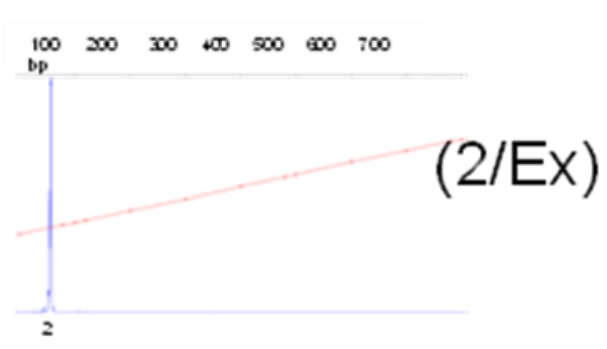

D

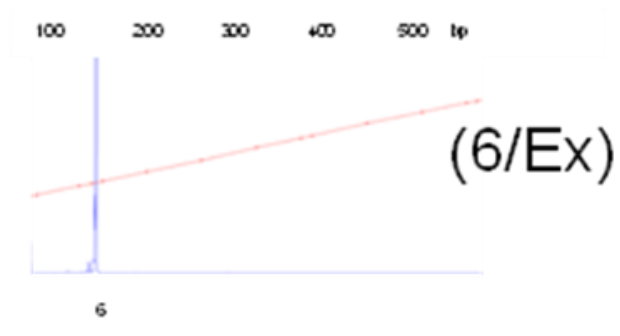

F

$100 \quad 200 \quad 300 \quad 40 \quad 500 \quad 600 \quad 200$ to

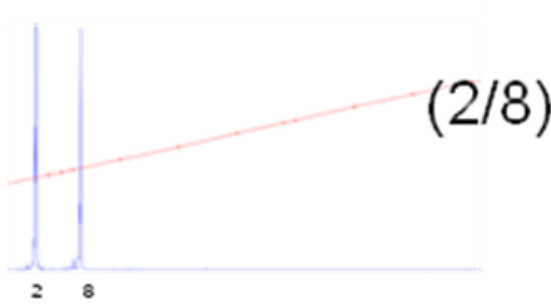

Supplementary Fig 1. 
A

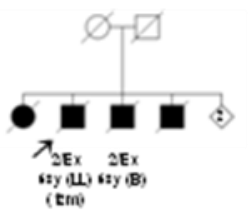

E

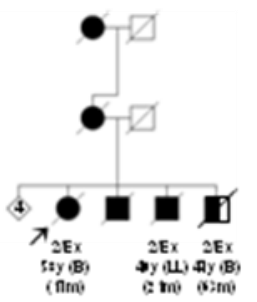

I

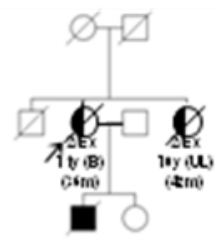

B

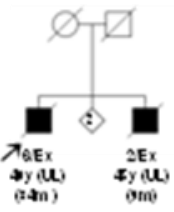

$\mathrm{F}$

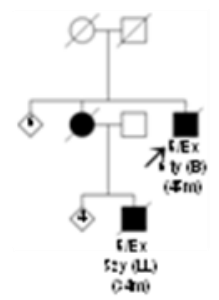

J
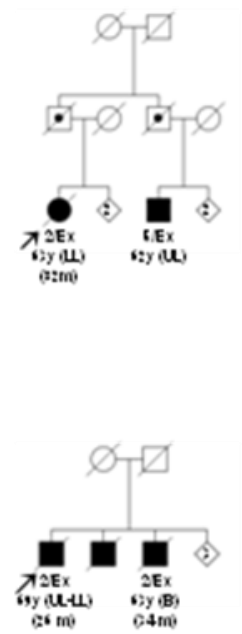

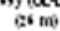

C

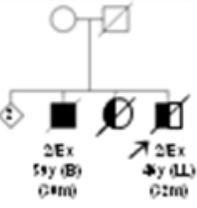

D

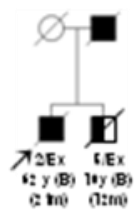

G

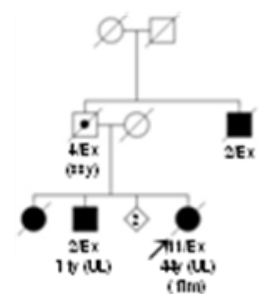

K

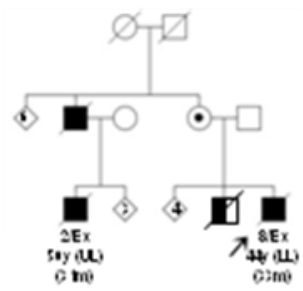

O

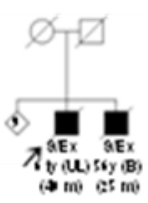

$\mathrm{H}$

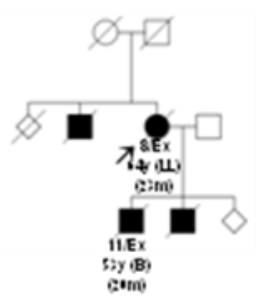

N

M

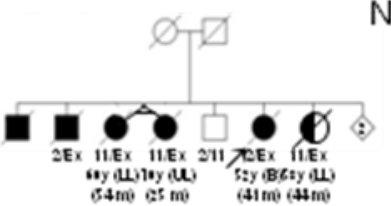

$$
\text { (1) }
$$$$
\text { (1) }
$$

$\mathrm{L}$

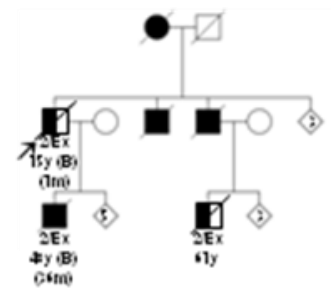

a

D $\mu, 0+10$

\section{Supplementary figure 2 . C9ORF72 repeat expansions segregate with the disease in 16}

\section{French pedigrees.}

Abbreviated pedigrees of families with C9ORF72 expanded repeats in which the segregation of the repeat could be studied (A-P). Arrows indicate index patients. Filled symbols indicate patients with ALS and half-filled symbols represent ALS patients with concomitant FTD. When available, the age at onset (years), site at onset ( $B=$ bulbar, UL=Upper Limb, LL=Lower Limb, in brackets) and the disease duration (in months, in brackets) are indicated below the symbol representing the patient. The number of repeats is indicated for each allele below the index case and some relatives for whom DNA was available. Ex ("expanded repeat") represents a number of repeats $>50$. The age at death ( $88 \mathrm{y})$ is indicated in brackets above an obligate carrier who did not develop the disease (G). 